TRANSACTIONS OF THE

AMERICAN MATHEMATICAL SOCIETY

Volume 363, Number 7, July 2011, Pages 3703-3716

S 0002-9947(2011)05249-9

Article electronically published on February 25, 2011

\title{
UNIQUE DECOMPOSITION INTO IDEALS FOR REDUCED COMMUTATIVE NOETHERIAN RINGS
}

\author{
BAŞAK AY AND LEE KLINGLER
}

\begin{abstract}
We say that a commutative ring $R$ has the unique decomposition into ideals (UDI) property if, for any $R$-module which decomposes into a finite direct sum of indecomposable ideals, this decomposition is unique up to the order and isomorphism class of the ideals. In a 2001 paper, Goeters and Olberding characterize the UDI property for Noetherian integral domains. In this paper, we characterize the UDI property for reduced Noetherian rings.
\end{abstract}

\section{INTRODUCTION}

The ring $R$ is said to have the unique decomposition into ideals (UDI) property if, for any $R$-module that decomposes into a finite direct sum of indecomposable ideals, this decomposition is unique apart from the order and isomorphism class of the ideals. In other words, for any indecomposable ideals $I_{1}, \ldots, I_{n}, J_{1}, \ldots, J_{m}$ of $R$, if $I_{1} \oplus \ldots \oplus I_{n} \cong J_{1} \oplus \ldots \oplus J_{m}$, then $n=m$ and, after reindexing, $I_{i} \cong J_{i}$, for each index $i$.

In the paper 4, Goeters and Olberding characterize the UDI property for Noetherian integral domains. In this paper, we give a characterization of the UDI property for reduced commutative Noetherian rings.

Let $R$ be a reduced commutative Noetherian ring; our goal is to determine necessary and sufficient conditions that $R$ have the UDI property. We remark that, if $R$ is isomorphic to $R_{1} \times R_{2}$, then the rings $R_{1}$ and $R_{2}$ are also reduced (commutative) Noetherian, and $R$ has the UDI property if and only if both $R_{1}$ and $R_{2}$ have the UDI property. Therefore, it suffices to characterize the UDI property in case $R$ is also assumed to be indecomposable. Thus, throughout this paper, $R$ will always denote an indecomposable, reduced, commutative, Noetherian ring.

We fix the following notation. The set of all prime ideals of $R$ is denoted by $\operatorname{Spec}(R)$, and the Zariski topology on $\operatorname{Spec}(R)$ has closed sets consisting of sets of the form $V(I)$, the set of all prime ideals containing $I$, as $I$ ranges over all ideals of $R$. Since $R$ is Noetherian, $\operatorname{Spec}(R)$ contains only finitely many minimal primes, which we denote by $P_{1}, \ldots, P_{t}$. If any of these minimal prime ideals, say $P_{1}$, is also a maximal ideal, then $V\left(P_{1}\right)=\left\{P_{1}\right\}$ would be both open and closed in $\operatorname{Spec}(R)$. Under the assumption that the $\operatorname{ring} R$ is indecomposable, the space $\operatorname{Spec}(R)$ is also indecomposable, so $\left\{P_{1}\right\}$ open and closed would make $\operatorname{Spec}(R)=\left\{P_{1}\right\}$; that is, $P_{1}$ would be the only prime ideal of $R$. Since we also assume that $R$ is reduced, this would force $P_{1}=0$ and $R$ to be a field. A field has the UDI property, so to avoid

Received by the editors March 18, 2009 and, in revised form, October 5, 2009 and November 13, 2009.

2010 Mathematics Subject Classification. Primary 13B21, 13B22, 13B30, $13 E 05$.

(c)2011 American Mathematical Society 3703

Reverts to public domain 28 years from publication 
this trivial case, we shall assume that none of the minimal prime ideals $P_{1}, \ldots, P_{t}$ is a maximal ideal. We remark that, since $R$ is assumed to be reduced, the set of zero-divisors of $R$ is precisely the set $P_{1} \cup \ldots \cup P_{t}$. Therefore, since the minimal prime ideals are all assumed to be non-maximal, it follows that no maximal ideal of $R$ is contained in the set of zero-divisors. Recall that an ideal is called regular if it contains a regular element. Thus, every maximal ideal of $R$ must be regular. Moreover, an ideal $I$ of $R$ is regular if and only if $A n n_{R}(I)=0$.

Let us fix $R_{i}=R / P_{i}$ for each index $i$. Because $R$ is reduced, $P_{1} \cap \ldots \cap P_{t}=0$, so the natural map $\phi: R \rightarrow R_{1} \times \ldots \times R_{t}$ is injective. We usually identify $R$ with its isomorphic image $\phi(R)$. Also let $R_{P_{i}}=Q_{i}=Q\left(R_{i}\right)$, the field of fractions of $R_{i}$, and let $\tilde{R}_{i}$ be the integral closure of $R_{i}$ in $Q_{i}$, for each index $i$. Then the total quotient ring of $R$ is the ring $Q=Q_{1} \times \ldots \times Q_{t}$, and the integral closure of $R$ is the ring $\tilde{R}=\tilde{R}_{1} \times \cdots \times \tilde{R}_{t}$.

We say that the $R$-module $G$ is torsion-free if no non-zero element is annihilated by a regular element of $R$. The rank of $G$ is the $t$-tuple $\operatorname{rank}(G)=\left(r_{1}, \ldots, r_{t}\right)$, where $r_{i}$ is the rank of the $Q_{i}$-vector-space $G_{P_{i}}$, for each index $i$.

This paper is organized as follows. In $\S 2$ we characterize the UDI property by showing that the ring $R$ has the UDI property if and only if either $R$ is a PID, or $R$ has a unique non-principal maximal ideal $M$, and $R_{M}$ has the UDI property. In $\S 3$ we give an explicit description of the local rings $R$ with the UDI property, building on the corresponding theorem for domains given in 4 . In $\S 4$ we provide some examples of rings which satisfy the UDI property, including a non-local domain of dimension two with the UDI property, and a non-local ring of arbitrary finite dimension $n$ with the UDI property.

We note the following well-known facts which we will find helpful throughout this paper. For completeness, we sketch the proofs.

Recall that, for ideals $I$ and $J$ of $R$, the colon ideal $[I: J]$ is defined to be $\{q \in Q: q J \subseteq I\}$.

Lemma 1.1. Let $I$ and $J$ be ideals of the ring $R$, with $J$ regular. Then the natural map from $[I: J]$ to $\operatorname{Hom}_{R}(J, I)$ is an isomorphism.

Proof. Let $\theta:[I: J] \rightarrow \operatorname{Hom}_{R}(J, I)$ be defined by $\theta(q)=\mu_{q}$, multiplication by $q$. Since $J$ is regular, clearly $\theta$ is injective. Let $\gamma \in \operatorname{Hom}_{R}(J, I)$, and fix a regular element $x \in J$. For any $y \in J, y \cdot \gamma(x)=\gamma(y x)=x \cdot \gamma(y)$, so that $\gamma=\theta\left(x^{-1} \gamma(x)\right)$, and hence $\theta$ is surjective.

Recall that a ring $R^{\prime}$ is called an overring of $R$ if $R \subseteq R^{\prime} \subseteq Q$.

Lemma 1.2. If $A$ and $B$ are torsion-free $R^{\prime}$-modules, where $R^{\prime}$ is an overring of $R$, then $\operatorname{Hom}_{R^{\prime}}(A, B)=\operatorname{Hom}_{R}(A, B)$.

Proof. Certainly any $R^{\prime}$-homomorphism is also an $R$-homomorphism. Conversely, suppose that $\phi: A \rightarrow B$ is an $R$-module homomorphism. Since $R \subseteq R^{\prime} \subseteq Q$, for any $s \in R^{\prime}$, we can write $s=x y^{-1}$ for some $x, y \in R$, with $y$ regular in $R$, so that $y s=x$. For each $a \in A$, since $s a \in A$, we get

$$
y s \phi(a)=x \phi(a)=\phi(x a)=\phi(y s a)=y \phi(s a) .
$$

Now, since $y$ is regular and $B$ is a torsion-free $R$-module, $s \phi(a)=\phi(s a)$, making $\phi$ an $R^{\prime}$-module homomorphism. 
Lemma 1.3. Let $P$ be a prime ideal of $R$ and $Q\left(R_{P}\right)$ the total quotient ring of $R_{P}$. Then $Q_{P} \subseteq Q\left(R_{P}\right)$.

Proof. Let $S_{1}$ be the set of all the regular elements of $R$ and $S_{2}=R-P$. Then $Q_{P}=S_{2}^{-1}\left(S_{1}^{-1} R\right)=\left(S_{1} S_{2}\right)^{-1} R=\left(\bar{S}_{1}\right)^{-1}\left(S_{2}^{-1} R\right)=\left(\bar{S}_{1}\right)^{-1}\left(R_{P}\right)$, where $\bar{S}_{1}$ denotes the image of $S_{1}$ in $R_{P}$. Since regular elements of $R$ remain regular in $R_{P}$, we get that $\bar{S}_{1}$ is contained in the set of regular elements of $R_{P}$, and hence $\left(\bar{S}_{1}\right)^{-1}\left(R_{P}\right) \subseteq$ $Q\left(R_{P}\right)$.

\section{Reduction to the Local CASE}

We remind the reader that, throughout this section, $R$ is assumed to be a reduced commutative Noetherian (indecomposable) ring but not a field. We begin with the key observation concerning the UDI property.

Proposition 2.1. If $R$ has the UDI property, then at most one maximal ideal of $R$ is non-principal.

Proof. Suppose that $M_{1}$ and $M_{2}$ are two distinct maximal ideals of $R$. The map $M_{1} \oplus M_{2} \rightarrow R$ is surjective and hence splits, so that $M_{1} \oplus M_{2} \cong R \oplus\left(M_{1} \cap M_{2}\right)$. Since $R$ is indecomposable and has the UDI property, $R$ must be isomorphic to a summand of $M_{1}$ or $M_{2}$, say $M_{1} \cong R t \oplus J$ for some regular element $t \in R$ and some ideal $J \subseteq R$. Then $R t \cap J=0$, implies that $t J=0$, and since $t$ is regular, it follows that $J=0$, so that $M_{1}$ is principal. Therefore, $R$ can have no more than one non-principal maximal ideal.

We can already see that the UDI property is not a local property. Indeed, if $F$ is a field, then the polynomial ring $F[X, Y]$ in two indeterminates certainly has more than one non-principal maximal ideal, so by Proposition [2.1, $F[X, Y]$ does not have the UDI property. On the other hand, since $F[X, Y]$ is integrally closed, the localization $F[X, Y]_{N}$ is integrally closed for each maximal ideal $N$ of $F[X, Y]$. Thus, the endomorphism ring of each non-zero ideal of $F[X, Y]_{N}$ must be $F[X, Y]_{N}$ itself, and hence by [1, Theorem I.3.6], $F[X, Y]_{N}$ has the UDI property for each maximal ideal $N$.

The goal of this section is to show that, in the presence of a unique non-principal maximal ideal, the UDI property is determined locally at that maximal ideal. First, we collect together some useful facts needed in the proof of this local reduction.

Lemma 2.2. The following hold for the ring $R$.

(1) For every principal maximal ideal $N \subseteq R$, the localization $R_{N}$ is a DVR, and $N$ properly contains a unique prime ideal, necessarily a minimal prime of $R$.

(2) If $R$ has no non-principal maximal ideal, then $R$ is a PID.

(3) If $R$ has a unique non-principal maximal ideal $M$, and if $a \in R$ is regular, then $a$ is contained in only finitely many maximal ideals of $R$.

(4) If $R$ has a unique non-principal maximal ideal $M$, then $M$ contains all the minimal prime ideals of $R$, so that $M$ contains all of the zero-divisors of $R$.

(5) If $R$ has a unique non-principal maximal ideal $M$, then $\operatorname{rank}(G)=\operatorname{rank}\left(G_{M}\right)$ for every finitely generated torsion-free $R$-module $G$.

Proof. (1) Since $R$ is reduced, $R_{N}$ must also be reduced. Thus, if $N$ is a principal maximal ideal of $R$, then $R_{N}$ is a local Noetherian ring with maximal ideal $N_{N}$ generated by a non-nilpotent element, so that $R_{N}$ is a DVR [8, Proposition I.2.2]. 
Therefore, the maximal ideal $N$ properly contains only one prime ideal, which must be a minimal prime of $R$.

(2) Suppose that all maximal ideals of $R$ are principal. From (1) it follows that each principal maximal ideal of $R$ contains a unique minimal prime ideal, so that $V\left(P_{1}\right)$ is an open and closed subset of $\operatorname{Spec}(R)$. Because $R$ is assumed to be indecomposable, the space $\operatorname{Spec}(R)$ is connected, and hence $P_{1}$ is the only minimal prime of $R$. Since $R$ is reduced, $P_{1}=0$, and hence $R$ is a domain. Because $R$ is a Noetherian domain and locally a DVR, $R$ is a Dedekind domain. Since all of the maximal ideals of $R$ are assumed to be principal, $R$ is a PID.

(3) Let $a$ be a regular element of $R$; it suffices to show that $a$ is contained in only finitely many principal maximal ideals of $R$. Let $a \in N$ for some principal maximal ideal $N \subseteq R$. Then $N$ contains a unique minimal prime ideal of $R$ by (1), and $a$ (being regular) cannot be an element of this minimal prime. Therefore, $N / R a$ is both a maximal ideal and a minimal prime ideal of the ring $R / R a$. Since $R / R a$ has only finitely many minimal prime ideals, $a$ can be contained in only finitely many principal maximal ideals of $R$.

(4) If a minimal prime ideal $P_{j}$ were not contained in $M$, then $V\left(P_{j}\right)$ would be an open and closed subset of $\operatorname{Spec}(R)$, so $R$ would be a PID by the argument given in the proof of (2). Since $R$ has a unique non-principal maximal ideal, it follows that each minimal prime ideal of $R$ must be contained in $M$. Moreover, since $P_{1} \cup \ldots \cup P_{t}$ is the set of all zero-divisors of $R$, it follows that $M$ contains all of the zero-divisors of $R$.

(5) By part (4), all minimal prime ideals are contained in $M$, so it follows that $\operatorname{rank}\left(\left(G_{M}\right)_{P}\right)=\operatorname{rank}\left(G_{P}\right)$ for each minimal prime $P$ of $R$, and hence $\operatorname{rank}\left(G_{M}\right)=$ $\operatorname{rank}(G)$.

We show that, in the presence of a unique non-principal maximal ideal, every ideal of $R$ not contained in this maximal ideal is principal. We use this to prove that the isomorphism class of a direct sum of ideals is determined locally at that maximal ideal, which is the main tool needed for the reduction to the local case.

Lemma 2.3. If $R$ has a unique non-principal maximal ideal $M$, then every ideal of $R$ not contained in $M$ is principal.

Proof. Suppose that $I \subseteq R$ is an ideal such that $I \nsubseteq M$, and let $a R$ be maximal among the principal ideals contained in $I$ but not in $M$. To show that $I=a R$, it suffices to prove equality locally at every maximal ideal of $R$. Since $a \notin M$, it follows that $R_{M}=a R_{M} \subseteq I_{M} \subseteq R_{M}$, and hence $a R_{M}=I_{M}$. Suppose, by way of a contradiction, that $a R_{N} \neq I_{N}$ for some principal maximal ideal $N=\pi R$ of $R$. Then $a R \subseteq N$ (else $R_{N}=a R_{N} \subseteq I_{N} \subseteq R_{N}$, as above), so that $a=\pi b$ for some $b \in R$. On the one hand, for a maximal ideal $P \neq N, \pi$ becomes a unit in $R_{P}$, and hence $b R_{P}=a R_{P} \subseteq I_{P}$. On the other hand, $R_{N}$ is a DVR, and $\pi b R_{N} \subsetneq I_{N}$, so that $b R_{N} \subseteq I_{N}$. It follows that $a R \subsetneq b R \subseteq I$, contrary to assumption. Therefore, $a R_{N}=I_{N}$ for every maximal ideal $N$ of $R$, as required.

Lemma 2.4. Suppose that $R$ has a unique non-principal maximal ideal $M$. Let $G$ and $H$ be finitely generated torsion-free modules, with $H$ isomorphic to a direct sum of indecomposable ideals of $R$. If $G_{M} \cong H_{M}$, then $G \cong H$.

Proof. Let us first assume that $H$ is an indecomposable ideal of $R$. Given that $G_{M} \cong H_{M}$, there exists a map $f: G \rightarrow H$ such that $f_{M}$ is an isomorphism. Then 
$\operatorname{Ker}\left(f_{M}\right)=(\operatorname{Ker}(f))_{M}=0$ implies that $s \cdot \operatorname{Ker}(f)=0$ for some $s \in R-M$. Since $s$ is regular by Lemma $2.2(4)$, and $\operatorname{Ker}(f)$ is torsion-free, it follows that $\operatorname{Ker}(f)=0$, and hence $f$ is injective. Set $I=\operatorname{Ann}_{R}(H / \operatorname{Im}(f))$, and note that $(H / \operatorname{Im}(f))_{M}=0$, so that $I \nsubseteq M$. By Lemma 2.3, $I=t R$ for some $t \in I-M$, so that $t$ is regular by Lemma $2.2(4)$.

We claim that $R / t R \cong H / t H$. By Lemma 2.2(3), $t$ is contained in only finitely many maximal ideals, all of which are principal. If $P$ is a maximal ideal such that $t \in P$, then $R_{P}$ is a DVR, and $t \in I$ implies that $H_{P} \neq 0$, so that $R_{P} \cong H_{P}$, and hence $R_{P} / t R_{P} \cong H_{P} / t H_{P}$. Thus, if we write $\bar{P}=P / t R$, then $(R / t R)_{\bar{P}} \cong(H / t H)_{\bar{P}}$ for each maximal ideal $\bar{P}$ of $R / t R$, and therefore $H / t H$ is a locally free $R / t R$-module of rank one. Since $R / t R$ is semi-local, $H / t H$ is free [6, Theorem 4.30], proving the claim.

From the above claim, and because $t H \subseteq \operatorname{Im}(f)$, there is a surjection $\alpha: R / t R \rightarrow$ $H / \operatorname{Im}(f)$. Since $t R=\operatorname{Ann}_{R}(H / \operatorname{Im}(f))$, it follows that $\operatorname{Ker}(\alpha)=t R$, and hence $H / t H \cong R / t R \cong H / \operatorname{Im}(f)$. We note that $R / t R$ is Artinian, so that $H / t H$ and $\mathrm{H} / \operatorname{Im}(f)$ have the same finite length. Then $t H \subseteq \operatorname{Im}(f)$ forces $\operatorname{Im}(f)=t H$, so that $G \cong \operatorname{Im}(f)=t H \cong H$.

In the general case, write $H=A \oplus B$, where $A$ is an indecomposable ideal of $R$. We claim that $G=A^{\prime} \oplus B^{\prime}$ for some submodules $A^{\prime}$ and $B^{\prime}$ such that $A_{M}^{\prime} \cong A_{M}$ and $B_{M}^{\prime} \cong B_{M}$. (It suffices to prove this claim, for it will then follow that $A^{\prime} \cong A$ by the first part of the proof, and $B^{\prime} \cong B$ by induction on the rank, so that the lemma follows.)

To prove the claim, let $f: G \rightarrow H$ such that $f_{M}$ is an isomorphism, and set $g=\pi \circ f$, where $\pi: A \oplus B \rightarrow A$ is the projection map. Let $A^{\prime \prime}=\operatorname{Im}(g) \subseteq A$. Since $f_{M}$ is an isomorphism and $\pi_{M}$ is surjective, it follows that $A_{M}^{\prime \prime}=(\operatorname{Im}(g))_{M}=$ $\operatorname{Im}\left(g_{M}\right)=A_{M}$, and hence $\operatorname{rank}\left(A^{\prime \prime}\right)=\operatorname{rank}\left(A_{M}^{\prime \prime}\right)=\operatorname{rank}\left(A_{M}\right)=\operatorname{rank}(A)$ by Lemma 2.2(5). For all principal maximal ideals $N \subseteq R$, the ring $R_{N}$ is a DVR (Lemma 2.2(1)), so that $A_{N}^{\prime \prime}$ and $A_{N}$ are free modules of the same rank and hence isomorphic; moreover, the map $g_{N}$ splits. On the other hand, $g_{M}=\pi_{M} \circ f_{M}$, where $f_{M}$ is an isomorphism and $\pi_{M}$ is a split surjection, so that the map $g_{M}$ also splits. Therefore, the map $g$ splits locally at every maximal ideal of $R$, and $A^{\prime \prime}=\operatorname{Im}(g)$ is finitely presented, from which it follows that the map $g$ splits. Thus, $G=A^{\prime} \oplus B^{\prime}$, where $A^{\prime} \cong \operatorname{Im}(g)=A^{\prime \prime}$ and $B^{\prime}=\operatorname{Ker}(g)$. Now $G_{M} \cong H_{M}$ by assumption, and $A_{M}^{\prime} \cong A_{M}^{\prime \prime} \cong A_{M}$ by construction, so that $B_{M}^{\prime} \cong B_{M}$ by local cancellation [2, Theorem 2], completing the proof of the claim.

We now have all of the tools needed to give a local characterization of the UDI property.

Theorem 2.5. $R$ has the UDI property if and only if $R$ is a PID or $R$ has a unique non-principal maximal ideal $M$, and $R_{M}$ has the UDI property.

Proof. If $R$ has the UDI property, then by Proposition 2.1 $R$ has at most one nonprincipal maximal ideal. If $R$ has no non-principal maximal ideal, then by Lemma 2.2(2), $R$ is a PID. Conversely, over a PID, finitely generated torsion-free modules are free. Hence it suffices to assume that $R$ has a unique non-principal maximal ideal $M$, and prove that $R$ has the UDI property if and only if $R_{M}$ has the UDI property.

Suppose that $R$ has the UDI property and that $A_{1}^{\prime}, \ldots, A_{t}^{\prime}, B_{1}^{\prime}, \ldots, B_{s}^{\prime}$ are indecomposable ideals of $R_{M}$ such that $A_{1}^{\prime} \oplus \ldots \oplus A_{t}^{\prime} \cong B_{1}^{\prime} \oplus \ldots \oplus B_{s}^{\prime}$. Set $A_{j}=A_{j}^{\prime} \cap R$ 
and $B_{i}=B_{i}^{\prime} \cap R$ for each index $j$ and each index $i$; since every ideal of $R_{M}$ is an extended ideal, we get that $A_{j}^{\prime}=A_{j} R_{M}$ and $B_{i}^{\prime}=B_{i} R_{M}$ for each index $j$ and each index $i$. Moreover, $A_{j}^{\prime}$ and $B_{i}^{\prime}$ indecomposable implies that $A_{j}$ and $B_{i}$ are also indecomposable for each index $i$ and each index $j$. Then $G=A_{1} \oplus \ldots \oplus A_{t}$ and $H=B_{1} \oplus \ldots \oplus B_{s}$ are finitely generated and torsion-free $R$-modules such that $G_{M} \cong H_{M}$. So, by Lemma2.4 it follows that $G \cong H$, and hence $t=s$, and $A_{j} \cong B_{j}$ for each index $j$ (reordering if necessary). Therefore, $A_{j}^{\prime} \cong B_{j}^{\prime}$ for each index $j$.

Conversely, suppose that $R_{M}$ has the UDI property, and suppose that $I_{1} \oplus \ldots \oplus$ $I_{n} \cong J_{1} \oplus \ldots \oplus J_{m}$ for some indecomposable ideals $I_{1}, \ldots, I_{n}, \ldots, J_{1}, \ldots, J_{m}$ of $R$. Note that, if $J$ is an indecomposable ideal of $R$, then $J_{M}$ must be an indecomposable ideal of $R_{M}$. For if $J_{M} \cong X^{\prime} \oplus Y^{\prime}$ for some ideals $X^{\prime}$ and $Y^{\prime}$ of $R_{M}$, then setting $X=R \cap X^{\prime}$ and $Y=R \cap Y^{\prime}$, as above we obtain $J_{M} \cong X_{M} \oplus Y_{M} \cong(X \oplus Y)_{M}$. From Lemma 2.4 it follows that $J \cong X \oplus Y$, so that $X=0$ or $Y=0$, and hence $X^{\prime}=0$ or $Y^{\prime}=0$, forcing $J_{M}$ to be indecomposable as well. Therefore, since each $\left(I_{i}\right)_{M}$ and $\left(J_{j}\right)_{M}$ is indecomposable as an $R_{M}$-module, and $R_{M}$ is assumed to have the UDI property, it follows that $n=m$, and reindexing if necessary, $\left(I_{j}\right)_{M} \cong\left(J_{j}\right)_{M}$ for each index $j$. By Lemma 2.4 again, $I_{j} \cong J_{j}$ for each index $j$.

\section{LOCAL RINGS WITH THE UDI PROPERTY}

Having reduced characterization of the UDI property for reduced commutative Noetherian rings to the case in which the ring is local, in this section we give a precise description of the reduced, commutative, Noetherian, local rings which have the UDI property. Thus, throughout this section, we assume that $R$ is a reduced, commutative, Noetherian, local ring with maximal ideal $M$. We begin by recalling a fundamental result [4, Theorem 3.2].

Theorem 3.1. Assume that $R$ is a local Noetherian domain with maximal ideal $M$, and let $\tilde{R}$ be the integral closure of $R$ in its field of fractions. Then $R$ has the UDI property if and only if there exists a fractional overring $R^{\prime}$ of $R$ with the same number of maximal ideals as $\tilde{R}$, such that one of the following holds.

(1) $R^{\prime}$ is local.

(2) $R^{\prime}$ has exactly two distinct maximal ideals $M_{1}^{\prime}, M_{2}^{\prime}$ such that $M_{1}^{\prime}$ is principal, with $M \nsubseteq\left(M_{1}^{\prime}\right)^{2}$ and $R^{\prime} / M_{1}^{\prime} \cong R / M$.

(3) $R^{\prime}=\tilde{R}$ has exactly three distinct maximal ideals $M_{1}^{\prime}, M_{2}^{\prime}, M_{3}^{\prime}$, such that all three are principal, with $M \nsubseteq\left(M_{j}^{\prime}\right)^{2}$ and $R^{\prime} / M_{j}^{\prime} \cong R / M$ for each index $j$.

Recall that $P_{1}, \ldots, P_{t}$ are the minimal prime ideals of our $\operatorname{ring} R$ and set $R_{i}=$ $R / P_{i}$ for each index $i$. We view $R \subseteq R_{1} \times \ldots \times R_{t}$ and let $Q=Q_{1} \times \ldots \times Q_{t}$ be the total quotient ring of $R$, where $Q_{i}$ is the field of fractions of $R_{i}$ for each index $i$. The integral closure of $R$ is written as $\tilde{R}=\tilde{R}_{1} \times \ldots \times \tilde{R}_{t}$, where $\tilde{R}_{i}$ is the integral closure of $R_{i}$ in $Q_{i}$ for each index $i$.

In order to characterize the UDI property in the case in which the local ring $R$ need not be a domain, we note a few cases in which UDI fails.

Lemma 3.2. If $t=2$ and $\tilde{R}_{2}$ has at least three distinct maximal ideals, then $R$ does not have the UDI property.

Proof. Given that $\tilde{R}_{2}$ has at least three distinct maximal ideals, we note that there exists a finitely generated overring $R_{2}^{\prime}$ of $R_{2}$ which has at least three distinct maximal ideals $M_{1}^{\prime}, M_{2}^{\prime}, M_{3}^{\prime}$, and we let $M_{1}$ be a maximal ideal of $R_{1}$. We observe that 
$R_{2}^{\prime}$ is a fractional ideal of $R$. Let $A=R+\left(M_{1} \times M_{1}^{\prime}\right)$ and $B=R_{2}+M_{2}^{\prime} M_{3}^{\prime}$; then $A$ and $B$ are finitely generated $R$-submodules of $Q$ and hence fractional ideals of $R$. Now, let us consider the map

$$
f: A \oplus B \rightarrow R_{2}^{\prime}
$$

defined by $f\left((r, s), s^{\prime}\right)=s+s^{\prime}$. The map $f$ is split by the map

$$
g: R_{2}^{\prime} \rightarrow A \oplus B
$$

defined by $g(t)=((0, t a), t b)$, for elements $a \in M_{1}^{\prime}$ and $b \in M_{2}^{\prime} M_{3}^{\prime}$ such that $a+b=1$. Thus, $\operatorname{Ker}(f) \oplus R_{2}^{\prime} \cong A \oplus B$.

Since $A$ and $B$ are multiplicatively closed, they are rings. In particular, $A=$ $\operatorname{End}_{A}(A)$. Since $A$ is an overring of $R$, it follows from Lemma 1.2 that $\operatorname{End}_{A}(A)=$ $\operatorname{End}_{R}(A)$, and hence $A=\operatorname{End}_{R}(A)$. Similarly, $B$ is an overring of $R_{2}=R / P_{2}$, so that $B=\operatorname{End}_{B}(B)=\operatorname{End}_{R_{2}}(B)=\operatorname{End}_{R}(B)$.

The non-trivial idempotent elements of $\tilde{R}$ are $(1,0)$ and $(0,1)$. Let $M_{2}=M_{1}^{\prime} \cap R_{2}$. If $(0,1) \in A$, then we could write $(0,1)=\left(r+m_{1}, s+m_{1}^{\prime}\right)$ for some elements $(r, s) \in R, m_{1} \in M_{1}$, and $m_{1}^{\prime} \in M_{1}^{\prime}$. Then $s+m_{1}^{\prime}=1$ implies that $s=1-m_{1}^{\prime} \notin M_{2}$, while $r+m_{1}=0$ implies that $r=-m_{1} \in M_{1}$. On the other hand, $R$ is local (with maximal ideal $M$ ) mapping onto the local rings $R_{1}$ and $R_{2}$ (with maximal ideals $M_{1}$ and $M_{2}$, respectively), so that either $(r, s) \in M$, in which case $r \in M_{1}$ and $s \in M_{2}$, or $(r, s) \notin M$, in which case $r \notin M_{1}$ and $s \notin M_{2}$. This contradiction shows that $(0,1) \notin A$. Since $(1,1) \in A$, but $(0,1) \notin A$, it follows that $(1,0) \notin A$, and hence $A$ is an indecomposable fractional ideal of $R$. Clearly $B$ is also an indecomposable fractional ideal of $R$.

By considering ranks, each of $A, B, R_{2}^{\prime}$, and $\operatorname{Ker}(f)$ is a fractional $R$-ideal, so if $R$ had the UDI property, comparison of ranks would force $R_{2}^{\prime} \cong B$ as $R$-modules, and hence as $R_{2}$-modules. We show that this leads to a contradiction, so that $R$ cannot have the UDI property.

We claim that $B$ is an $R_{2}^{\prime}$-module. Since $R_{2} \subseteq Q_{2}$, for any $s^{\prime} \in R_{2}^{\prime}$ we can write $s^{\prime}=r_{1} s_{1}^{-1}$ for some $r_{1}, s_{1} \in R_{2}$. Let $\theta: R_{2}^{\prime} \rightarrow B$ be an $R_{2}$-module isomorphism, and for $b \in B$, write $b=\theta\left(r^{\prime}\right)$ for some $r^{\prime} \in R_{2}^{\prime}$. Since $R_{2}$ is a domain, it follows that

$$
s_{1} s^{\prime} b=r_{1} \theta\left(r^{\prime}\right)=\theta\left(r_{1} r^{\prime}\right)=\theta\left(s_{1} s^{\prime} r^{\prime}\right)=s_{1} \theta\left(s^{\prime} r^{\prime}\right),
$$

so that $s^{\prime} b=\theta\left(s^{\prime} r^{\prime}\right) \in B$. Thus, $B$ is an $R_{2}^{\prime}$-module, so that $R_{2}^{\prime}$ and $B$ are isomorphic as $R_{2}^{\prime}$-modules by Lemma 1.2, and hence $B$ is an ideal of $R_{2}^{\prime}$. But $B$ is a ring, so that $1 \in B$, and therefore $B=R_{2}^{\prime}$. Since $M_{2} \subseteq M_{2}^{\prime} \cap M_{3}^{\prime}=M_{2}^{\prime} M_{3}^{\prime}$, where $M_{2}=M / P_{2}$ is maximal, it follows that $M_{2}=M_{2}^{\prime} M_{3}^{\prime} \cap R_{2}$, so that

$$
R_{2}^{\prime} / M_{2}^{\prime} M_{3}^{\prime}=\left(R_{2}+M_{2}^{\prime} M_{3}^{\prime}\right) / M_{2}^{\prime} M_{3}^{\prime} \cong R_{2} / M_{2},
$$

contradicting the fact that $M_{2}^{\prime} M_{3}^{\prime}$ is not a prime ideal in $R_{2}^{\prime}$. Therefore, $R$ cannot have the UDI property.

Lemma 3.3. If $t=2$ and $\tilde{R}_{1}$ and $\tilde{R}_{2}$ each has at least two distinct maximal ideals, then $R$ does not have the UDI property.

Proof. Given that each of the integral closures $\tilde{R}_{1}$ and $\tilde{R}_{2}$ has at least two distinct maximal ideals, as above there exist finitely generated overrings $R_{1}^{\prime}$ of $R_{1}$ and $R_{2}^{\prime}$ of $R_{2}$, with distinct maximal ideals $M_{1}^{\prime}, M_{2}^{\prime}$ and $N_{1}^{\prime}, N_{2}^{\prime}$, respectively. Also as above, $R_{1}^{\prime}$ and $R_{2}^{\prime}$ are fractional ideals of $R$. Let us consider the map

$$
f:\left(R+M_{1}^{\prime} \times N_{1}^{\prime}\right) \oplus\left(R+M_{2}^{\prime} \times N_{2}^{\prime}\right) \rightarrow R_{1}^{\prime} \oplus R_{2}^{\prime}
$$


defined by $f\left((r, s),\left(r^{\prime}, s^{\prime}\right)\right)=\left(r+r^{\prime}, s+s^{\prime}\right)$. The map $f$ is split by the map

$$
g: R_{1}^{\prime} \oplus R_{2}^{\prime} \rightarrow\left(R+M_{1}^{\prime} \times N_{1}^{\prime}\right) \oplus\left(R+M_{2}^{\prime} \times N_{2}^{\prime}\right)
$$

defined by $g\left(t, t^{\prime}\right)=\left(\left(t a, t^{\prime} b\right),\left(t a^{\prime}, t^{\prime} b^{\prime}\right)\right)$, for elements $a \in M_{1}^{\prime}$ and $a^{\prime} \in M_{2}^{\prime}$ such that $a+a^{\prime}=1$, and $b \in N_{1}^{\prime}$ and $b^{\prime} \in N_{2}^{\prime}$ such that $b+b^{\prime}=1$.

Therefore, $\operatorname{Ker}(f) \oplus R_{1}^{\prime} \oplus R_{2}^{\prime} \cong\left(R+M_{1}^{\prime} \times N_{1}^{\prime}\right) \oplus\left(R+M_{2}^{\prime} \times N_{2}^{\prime}\right)$. Since the rank of $\operatorname{Ker}(f)$ is at most 1 at each minimal prime ideal, it is a fractional ideal of $R$. By a similar argument as in Lemma 3.2. $R+M_{1}^{\prime} \times N_{1}^{\prime}$ and $R+M_{2}^{\prime} \times N_{2}^{\prime}$ are indecomposable fractional ideals of $R$. But their ranks are different from the rank of $R_{1}^{\prime}$, so it follows that $R$ cannot have the UDI property.

Lemma 3.4. If $t \geq 3$ and $\tilde{R}_{1}$ has at least two distinct maximal ideals, then $R$ does not have the UDI property.

Proof. Let $\nu_{1}: R \rightarrow R_{1} \times R_{2}$ and $\nu_{2}: R \rightarrow R_{1} \times R_{3}$ be the projection maps, with $R^{\prime}=\operatorname{Im}\left(\nu_{1}\right)$ and $R^{\prime \prime}=\operatorname{Im}\left(\nu_{2}\right)$. There exists a finitely generated overring $R_{1}^{\prime}$ of $R_{1}$ with at least two distinct maximal ideals $M_{1}^{\prime}$ and $M_{2}^{\prime}$; let $M_{2}$ and $M_{3}$ be the maximal ideals of $R_{2}$ and $R_{3}$, respectively. As above, $R_{1}^{\prime}$ is a fractional ideal of $R$. Now, let us consider the map

$$
f:\left(R^{\prime}+M_{1}^{\prime} \times M_{2}\right) \oplus\left(R^{\prime \prime}+M_{2}^{\prime} \times M_{3}\right) \rightarrow R_{1}^{\prime}
$$

defined by $f\left((r, s),\left(r^{\prime}, s^{\prime}\right)\right)=r+r^{\prime}$. The map $f$ is split by the map

$$
g: R_{1}^{\prime} \rightarrow\left(R^{\prime}+M_{1}^{\prime} \times M_{2}\right) \oplus\left(R^{\prime \prime}+M_{2}^{\prime} \times M_{3}\right)
$$

defined by $g(t)=\left((t a, 0),\left(t a^{\prime}, 0\right)\right)$, for elements $a \in M_{1}^{\prime}$ and $a^{\prime} \in M_{2}^{\prime}$ such that $a+a^{\prime}=1$. It follows that $\operatorname{Ker}(f) \oplus R_{1}^{\prime} \cong\left(R^{\prime}+M_{1}^{\prime} \times M_{2}\right) \oplus\left(R^{\prime \prime}+M_{2}^{\prime} \times M_{3}\right)$, and comparing ranks shows that $\operatorname{Ker}(f)$ is a fractional ideal of $R$. By a similar argument as in Lemma 3.2, one can show that $R^{\prime}+M_{1}^{\prime} \times M_{2}$ and $R^{\prime \prime}+M_{2}^{\prime} \times M_{3}$ are indecomposable fractional ideals of $R$, and their ranks are different from that of $R_{1}^{\prime}$, so that $R$ fails to have the UDI property.

The next lemma will allow us to replace the ring $R$ by a homomorphic image in which a given ideal becomes regular.

Lemma 3.5. Suppose that $R$ has a unique non-principal maximal ideal $M$, and let $J$ be a non-zero non-regular indecomposable ideal of $R$. Let $\psi: R \rightarrow R / \operatorname{Ann}_{R}(J)$ be the natural map, and set $\bar{R}=R / \operatorname{Ann}_{R}(J), \bar{M}=\psi(M)=\left(M+\operatorname{Ann}_{R}(J)\right) / \operatorname{Ann}_{R}(J)$, and $\bar{J}=\left(J+\operatorname{Ann}_{R}(J)\right) / \operatorname{Ann}_{R}(J)$. The following all hold.

(1) $\operatorname{Ann}_{R}(J) \subseteq M$, so that $\bar{M}=M / \operatorname{Ann}_{R}(J)$.

(2) $\bar{R}$ is reduced.

(3) $\bar{J} \cong J$ as $R$-modules.

(4) $\bar{J}$ is an indecomposable regular ideal of $\bar{R}$.

Proof. (1) Since $\operatorname{Ann}_{R}(J)$ consists of zero-divisors and $M$ contains all the zerodivisors of $R, \operatorname{Ann}_{R}(J) \subseteq M$.

(2) If $r^{n} \in \operatorname{Ann}_{R}(J)$, for some $n$, then $r^{n} J^{n}=(r J)^{n}=0$, which implies $r J=0$, since $R$ is reduced, so $r \in \operatorname{Ann}_{R}(J)$.

(3) Since $R$ is reduced, $J \cap \operatorname{Ann}_{R}(J)=0$, implying that $\bar{J}=\psi(J)=(J+$ $\left.\operatorname{Ann}_{R}(J)\right) / \operatorname{Ann}_{R}(J) \cong J /\left(\operatorname{Ann}_{R}(J) \cap J\right) \cong J$ as $R$-modules.

(4) Let $\bar{x} \in \bar{R}$ such that $\bar{x} \bar{J}=0$. Then $x J \subseteq \operatorname{Ann}_{R}(J)$, so $x J \cdot J=0$ implies that $x J=0$, since $R$ is reduced. Thus, $x \in \operatorname{Ann}_{R}(J)$, and hence $\bar{x}=0$; that 
is, $\bar{J}$ is regular. Since $\bar{J} \cong J$ and $J$ is indecomposable, it follows that $\bar{J}$ is also indecomposable.

Theorem 3.6. Let $R$ be a reduced, commutative, Noetherian, local ring with maximal ideal $M$. Then $R$ has the UDI property if and only if one of the following holds.

(1) $t=1$, and $R$ satisfies one of the conditions of Theorem 3.1 .

(2) $t=2, \tilde{R}_{1}$ is local with the maximal ideal $\tilde{N}$, and $\tilde{R}_{2}$ is finite over $R_{2}$ and a PID with exactly two distinct maximal ideals $\tilde{M}_{1}$ and $\tilde{M}_{2}$, such that $M \nsubseteq \tilde{R}_{1} \times\left(\tilde{M}_{i}\right)^{2}$ and $\tilde{R}_{2} / \tilde{M}_{i} \cong R / M$ for $i=1,2$.

(3) $t \geqslant 2$, and $\tilde{R}_{i}$ is local for all $i, 1 \leqslant i \leqslant t$.

Proof. Suppose first that $R$ has the UDI property. If $t=1$, then (1) holds by Theorem 3.1] so we can suppose that $t \geq 2$. If every $\tilde{R}_{i}$ is local, then (3) holds, so we can suppose also that at least one $\tilde{R}_{i}$ is non-local. By Lemma 3.4, it must be the case that $t=2$. By Lemma 3.3, one of $\tilde{R}_{1}$ or $\tilde{R}_{2}$ must be local, say $\tilde{R}_{1}$. By Lemma 3.2, the ring $\tilde{R}_{2}$ must have exactly two distinct maximal ideals $\tilde{M}_{1}$ and $\tilde{M}_{2}$. As noted above, there exists a finite overring $R_{2}^{\prime}$ of $R_{2}$, such that $R_{2}^{\prime}$ has exactly two distinct maximal ideals $M_{1}^{\prime}$ and $M_{2}^{\prime}$, where $M_{i}^{\prime}=R_{2}^{\prime} \cap \tilde{M}_{i}$ for each index $i$.

Suppose that $X$ is an $R_{2}$-submodule of $R_{2}^{\prime}$ such that $\left(M_{2}^{\prime}\right)^{2} \subseteq X$, so that $m_{1}+x=$ 1 for some $m_{1} \in M_{1}^{\prime}$ and $x \in\left(M_{2}^{\prime}\right)^{2} \subseteq X$. We observe that $X$ is a fractional ideal of the integral domain $R_{2}$, so that $X$ is an indecomposable fractional $R$-module. Then consider the homomorphism

$$
f:\left(R+M_{1}^{\prime}\right) \oplus X \rightarrow R_{2}^{\prime}
$$

defined by $f\left((s, r), r^{\prime}\right)=r+r^{\prime}$. The map $f$ is split by the map

$$
g: R_{2}^{\prime} \rightarrow\left(R+M_{1}^{\prime}\right) \oplus X
$$

defined by $g(r)=\left(\left(0, m_{1} r\right), x r\right)$, so that $\operatorname{Ker}(f) \oplus R_{2}^{\prime} \cong\left(R+M_{1}^{\prime}\right) \oplus X$. By a similar argument as in Lemma 3.2, we note that $R+M_{1}^{\prime}$ is an overring of $R$ as well as an indecomposable fractional ideal of $R$. Furthermore, $R_{2}^{\prime}$ is a fractional ideal of $R$, because $R_{2}^{\prime}$ is a finitely generated $R_{2}$-submodule of $Q_{2}$ and hence $R$-submodule of $Q$. Moreover, by considering ranks, $\operatorname{Ker}(f)$ must be a fractional ideal of $R$ as well. Since $R$ has the UDI property, by comparing ranks, we see that $X \cong R_{2}^{\prime}$ as $R$-modules and hence as $R_{2}$-modules. As shown in Lemma 3.2, this forces $X$ to be an $R_{2}^{\prime}$-module as well, so that $X=y R_{2}^{\prime}$ for some $y \in X$. We shall apply this construction several times.

First, by choosing $X=M_{2}^{\prime}$, we see that $M_{2}^{\prime}$ must be a principal ideal of $R_{2}^{\prime}$. Reversing the roles of $M_{1}^{\prime}$ and $M_{2}^{\prime}$, we see that $M_{1}^{\prime}$ is also principal, and hence $R_{2}^{\prime}$ is a PID. Since $R_{2}^{\prime}$ is integrally closed, it follows that $R_{2}^{\prime}=\tilde{R}_{2}$.

Next, for any $R_{2}$-module $X$ such that $M_{2}^{\prime} \subseteq X \subsetneq R_{2}^{\prime}$, we see that $X$ must be an ideal of $R_{2}^{\prime}$, so that, by the maximality of $M_{2}^{\prime}, X=M_{2}^{\prime}$. Therefore, $R_{2}^{\prime} / M_{2}^{\prime}$ is a simple $R_{2}$-module, so it is a one-dimensional vector space over $R_{2} / M_{2}$, where $M_{2}=M_{2}^{\prime} \cap R_{2}$. Thus, the inclusion $R_{2} \hookrightarrow R_{2}^{\prime}$ induces an isomorphism $R_{2} / M_{2} \cong$ $R_{2}^{\prime} / M_{2}^{\prime}$, and hence $R_{2}^{\prime} / M_{2}^{\prime} \cong R_{2} / M_{2} \cong R / M$. Similarly, $R_{2}^{\prime} / M_{1}^{\prime} \cong R / M$.

Finally, we can choose $X=R_{2}+\left(M_{2}^{\prime}\right)^{2}$, an $R_{2}$-submodule of $R_{2}^{\prime}$. Then since $1 \in X$, and $X$ is an ideal of $R_{2}^{\prime}$, it follows that $X=R_{2}^{\prime}$. Let us write $M_{2}^{\prime}=\pi R_{2}^{\prime}$ for some $\pi \in M_{2}^{\prime}$. Then $\pi \in X$ implies that $\pi=r+s \pi^{2}$ for some $r \in R_{2}$ and $s \in R_{2}^{\prime}$, so that $r=\pi-s \pi^{2} \in R_{2} \cap M_{2}^{\prime}=M_{2}$. Since $\pi \notin R_{2}^{\prime} \pi^{2}$, it follows that $r \notin\left(M_{2}^{\prime}\right)^{2}$, and hence $M \nsubseteq \tilde{R}_{1} \times\left(M_{2}^{\prime}\right)^{2}$. Similarly, $M \nsubseteq \tilde{R}_{1} \times\left(M_{1}^{\prime}\right)^{2}$. 
Conversely, let us suppose that one of (1), (2) or (3) holds. We consider each case in turn.

If (1) holds, then by Theorem 3.1, $R$ has the UDI property.

Suppose instead that $(2)$ holds, and that $I_{1} \oplus \ldots \oplus I_{n} \cong J_{1} \oplus \ldots \oplus J_{m}$ for some indecomposable ideals $I_{1}, \ldots, I_{n}, J_{1}, \ldots, J_{m}$ of $R$. If some $\operatorname{End}_{R}\left(I_{k}\right)$ is local, then by [1, Lemma I.3.4], $I_{k} \cong J_{l}$ for some index $l$, so by local cancellation [2, Theorem $2]$, we can cancel $I_{k}$ and $J_{l}$ from the isomorphism. Thus, by induction, we can assume that each endomorphism $\operatorname{ring} \operatorname{End}_{R}\left(I_{k}\right)$ and $\operatorname{End}_{R}\left(J_{l}\right)$ is non-local.

Note that, if $I$ is a non-zero ideal of $R$, then $\operatorname{rank}(I)$ must be $(1,0),(0,1)$, or $(1,1)$. If $\operatorname{rank}(I)=(1,0)$, then $\operatorname{Ann}_{R}(I)=P_{1}$, and $I \cong \bar{I}=\left(I+P_{1}\right) / P_{1}$ is an ideal of $\bar{R}=R / P_{1}=R_{1}$. Hence $\operatorname{End}_{R}(I) \cong \operatorname{End}_{\bar{R}}(\bar{I})$ is a finite overring of $R_{1}$, which makes it local, since $\tilde{R}_{1}$ is local. Thus, we assume that none of the remaining summands $I_{k}$ and $J_{l}$ has rank $(1,0)$.

On the other hand, if $\operatorname{rank}(I)=(0,1)$, then $\operatorname{Ann}_{R}(I)=P_{2}$, and as above, $I \cong \bar{I}=\left(I+P_{2}\right) / P_{2}$ is an ideal of $\bar{R}=R / P_{2}=R_{2}$. Let $R^{\prime}=\operatorname{End}_{\bar{R}}(\bar{I}) \cong \operatorname{End}_{R}(I)$, so that $R_{2} \subseteq R^{\prime} \subseteq \tilde{R}_{2}$; we claim that $R^{\prime}=\tilde{R}_{2}$. Let $M_{i}^{\prime}=R^{\prime} \cap \tilde{M}_{i}$ for $i=1,2$, so that $M_{1}^{\prime} \neq M_{2}^{\prime}$, since $R^{\prime}$ is assumed to be non-local. For each index $i, R / M=$ $R_{2} / M_{2} \subseteq R^{\prime} / M_{i}^{\prime} \subseteq \tilde{R}_{2} / \tilde{M}_{i} \cong R / M$ implies that $\tilde{R}_{2}=R^{\prime}+\tilde{M}_{i}$. Moreover, since $M \nsubseteq\left(\tilde{R}_{1} \times \tilde{M}_{i}\right)^{2}$, it follows that $M_{i}^{\prime} \nsubseteq \tilde{M}_{i}^{2}$. We note that since $\tilde{R}_{2}$ is a PID with exactly two maximal ideals, each $\left(\tilde{R}_{2}\right)_{M_{i}^{\prime}}$ is a DVR, and $M_{i}^{\prime}\left(\tilde{R}_{2}\right)_{M_{i}^{\prime}} \nsubseteq\left(\tilde{M}_{i}\right)_{M_{i}^{\prime}}^{2}$. Thus, $\left(\tilde{M}_{i}\right)_{M_{i}^{\prime}}=M_{i}^{\prime}\left(\tilde{R}_{2}\right)_{M_{i}^{\prime}}$, and hence $\left(\tilde{R}_{2}\right)_{M_{i}^{\prime}}=R_{M_{i}^{\prime}}^{\prime}+M_{i}^{\prime}\left(\tilde{R}_{2}\right)_{M_{i}^{\prime}}$. Therefore, by Nakayama's Lemma, since $\tilde{R}_{2}$ is finitely generated as an $R_{2}$-module, $R_{M_{i}^{\prime}}^{\prime}=\left(\tilde{R}_{2}\right)_{M_{i}^{\prime}}$ for each index $i$, from which it follows that $R^{\prime}=\tilde{R}_{2}$, as claimed. Thus $I \cong \bar{I}$, an ideal of the PID $\tilde{R}_{2}$, so that $I \cong \tilde{R}_{2}$ as $\tilde{R}_{2}$-modules, and hence as $R$-modules.

In the direct sums $I_{1} \oplus \ldots \oplus I_{n} \cong J_{1} \oplus \ldots \oplus J_{m}$, we assume that each indecomposable summand has rank $(0,1)$ or $(1,1)$ and has a non-local endomorphism ring. Since the sums of the ranks are equal on both sides, there are the same number of ideals of rank $(0,1)$ on both sides. We just showed that each such ideal is isomorphic to $\tilde{R}_{2}$ as an $R$-module, so by local cancellation [2, Theorem 2], we can cancel those summands from both sides of the isomorphism. Therefore, we can further assume that each $I_{k}$ and each $J_{l}$ has rank $(1,1)$ and hence is regular.

Let us fix the indecomposable regular ideal $I=I_{1}$ with non-local endomorphism ring $R^{\prime}=\operatorname{End}_{R}(I)$. The ring $\tilde{R}=\tilde{R}_{1} \times \tilde{R}_{2}$ has exactly three maximal ideals, $\tilde{N} \times \tilde{R}_{2}, \tilde{R}_{1} \times \tilde{M}_{1}$, and $\tilde{R}_{1} \times \tilde{M}_{2}$. Let $N^{\prime}=R^{\prime} \cap\left(\tilde{N} \times \tilde{R}_{2}\right), M_{i}^{\prime}=R^{\prime} \cap\left(\tilde{R}_{1} \times \tilde{M}_{i}\right)$, for $i=1,2$. We claim that $N^{\prime}=M_{1}^{\prime}$ or $N^{\prime}=M_{2}^{\prime}$ (but, since $R^{\prime}$ is non-local, not both). Let us assume, by the way of contradiction, that $N^{\prime} \neq M_{1}^{\prime}$ and $N^{\prime} \neq M_{2}^{\prime}$. The ring $\tilde{R}$ has only two minimal primes, $\tilde{P}_{1}=\{0\} \times \tilde{R}_{2}$ and $\tilde{P}_{2}=\tilde{R}_{1} \times\{0\}$, so that $\tilde{R}_{i}=\tilde{R} / \tilde{P}_{i}$ for each index $i$. Let $P_{i}^{\prime}=R^{\prime} \cap \tilde{P}_{i}$ for each index $i$, so that $P_{1}^{\prime}$ and $P_{2}^{\prime}$ are the (distinct) minimal primes of $R^{\prime}$. Now $\tilde{P}_{1}$ is contained in only one maximal ideal, $\tilde{N} \times \tilde{R}_{2}$, so $P_{1}^{\prime} \subseteq N^{\prime}$. Similarly, $\tilde{P}_{2}$ is contained in two distinct maximal ideals $\tilde{R}_{1} \times \tilde{M}_{1}$ and $\tilde{R}_{1} \times \tilde{M}_{2}$, so that $P_{2}^{\prime} \subseteq M_{1}^{\prime}, M_{2}^{\prime}$. Since $\tilde{R}$ is integral over $R^{\prime}$, it follows by "going-up" that $N^{\prime}$ is the only maximal ideal of $R^{\prime}$ containing $P_{1}^{\prime}$, and $M_{1}^{\prime}$ and $M_{2}^{\prime}$ are the only maximal ideals of $R^{\prime}$ containing $P_{2}^{\prime}$. Thus, $\operatorname{Spec}\left(R^{\prime}\right)$ decomposes as the disjoint union of $V\left(P_{1}^{\prime}\right)$ and $V\left(P_{2}^{\prime}\right)$, which makes $R^{\prime}$ decomposable, and hence $I$ would be decomposable, contrary to assumption. This proves the claim, so we can assume that $N^{\prime}=M_{1}^{\prime} \neq M_{2}^{\prime}$. As argued above, one can show that 
$\left(\tilde{M}_{2}\right)_{M_{2}^{\prime}}=\left(M_{2}^{\prime} \tilde{R}_{2}\right)_{M_{2}^{\prime}}$. Moreover, $\left(\tilde{R}_{2}\right)_{M_{2}^{\prime}}$ is a finitely generated $R_{M_{2}^{\prime}}^{\prime}$-module, since $\tilde{R}_{2}$ is finite over $R_{2}$, so by Nakayama's Lemma, $\left(\tilde{R}_{2}\right)_{M_{2}^{\prime}}=R_{M_{2}^{\prime}}^{\prime}$. Therefore, $R_{M_{2}^{\prime}}^{\prime}$ is a DVR, so that $M_{2}^{\prime} R_{M_{2}^{\prime}}^{\prime}$ is principal.

Without loss of generality, we can assume that $R^{\prime}=\operatorname{End}_{R}\left(I_{1}\right)$ is minimal with

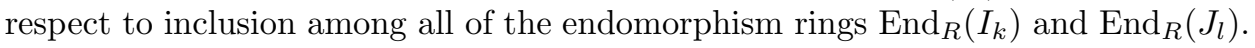
Let us regard $L=I_{1} \oplus \ldots \oplus I_{n}=J_{1} \oplus \ldots \oplus J_{n}$ as two internal decompositions of $L$ into direct sums of ideals. Now using the inclusion maps $\iota_{j}: I_{j} \rightarrow L$ and $\iota_{j}^{\prime}: J_{j} \rightarrow L$ and the projection maps $\pi_{j}: L \rightarrow I_{j}$ and $\pi_{j}^{\prime}: L \rightarrow J_{j}$, the identity map on $I_{1}$ is $\pi_{1} \iota_{1}=\sum_{j} \pi_{1} \iota_{j}^{\prime} \pi_{j}^{\prime} \iota_{1}$, where each composition $\pi_{1} \iota_{j}^{\prime} \pi_{j}^{\prime} \iota_{1}$ is in $R^{\prime}$. Since $R_{M_{1}^{\prime}}^{\prime}$ is a local ring, one of these compositions must be a unit in $R_{M_{1}^{\prime}}^{\prime}$; we can assume that it is $\pi_{1} \iota_{1}^{\prime} \pi_{1}^{\prime} \iota_{1}$. Let $f=\pi_{1} \iota_{1}^{\prime}$, an $R$-homomorphism from $J_{1}$ to $I_{1}$; by assumption $f\left(J_{1}\right) R_{M_{1}^{\prime}}^{\prime}=\left(I_{1}\right)_{M_{1}^{\prime}}$. Using the fact that $M_{1}^{\prime} \neq M_{2}^{\prime}$, we can choose $a \in M_{2}^{\prime}-\left(M_{1}^{\prime} \cup P_{2}^{\prime} \cup\left(M_{2}^{\prime}\right)^{2}\right)$, which makes a regular. Since $R_{M_{2}^{\prime}}^{\prime}$ is a DVR, there exists $n \geqslant 0$ such that $f\left(J_{1}\right) R_{M_{2}^{\prime}}^{\prime}=a^{n}\left(I_{1}\right)_{M_{2}^{\prime}}$. Now $a$ is regular, so we can set $h=a^{-n} f$, which is a $Q$-endomorphism of $Q$. Since $a$ is a unit in $R_{M_{1}^{\prime}}^{\prime}, h\left(J_{1}\right) R_{M_{1}^{\prime}}^{\prime}=$ $f\left(J_{1}\right) R_{M_{1}^{\prime}}^{\prime}=\left(I_{1}\right)_{M_{1}^{\prime}}$, while $h\left(J_{1}\right) R_{M_{2}^{\prime}}^{\prime}=\left(I_{1}\right)_{M_{2}^{\prime}}$ by choice of $n$. Then $h\left(J_{1}\right) R^{\prime}$ and $I_{1}$ are locally equal at both maximal ideals of $R^{\prime}$, so that $h\left(J_{1}\right) R^{\prime}=I_{1}$. We can view $\operatorname{Hom}_{R}\left(J_{1}, I_{1}\right)$ as an $R$-submodule of $Q$, since $I_{1}$ and $J_{1}$ are regular (Lemma 1.1), so let $U=\operatorname{Hom}_{R}\left(J_{1}, I_{1}\right) J_{1}$, an $R^{\prime}$-submodule of $I_{1}$. Since $h \in \operatorname{Hom}_{R}\left(J_{1}, I_{1}\right)$ and $h\left(J_{1}\right) \subseteq U$, it follows that $U=I_{1}$. If we set $R^{\prime \prime}=\operatorname{End}_{R}\left(J_{1}\right)$, then $U$ is an $R^{\prime \prime}$-module, which makes $I_{1}$ an $R^{\prime \prime}$-module, so that $R^{\prime \prime} \subseteq R^{\prime}$. By minimality of $R^{\prime}$, it follows that $R^{\prime}=R^{\prime \prime}$. Thus, by Lemma 1.2, $I_{1}=h\left(J_{1}\right) R^{\prime}=h\left(J_{1} R^{\prime}\right)=h\left(J_{1}\right)$, which makes $h$ surjective as an $R^{\prime}$-map, so as an $R$-map, again by Lemma 1.2 . The ranks are equal, so $h$ must also be injective, and hence $h: J_{1} \rightarrow I_{1}$ is an $R$-isomorphism. Therefore, by local cancellation [2, Theorem 2] and induction, it follows that $R$ has the UDI property.

Finally, let us suppose that (3) holds. We claim that every indecomposable ideal of $R$ has a local endomorphism ring. Let $I$ be an indecomposable ideal of $R$, and set $\bar{R}=R / \operatorname{Ann}_{R}(I)$, and $\bar{I}=\left(I+\operatorname{Ann}_{R}(I)\right) / \operatorname{Ann}_{R}(I)$, a regular ideal of $\bar{R}$. If $I$ is nonregular, then by Lemma $3.5(3), I \cong \bar{I}$ as $R$-modules, so that $\operatorname{End}_{R}(I) \cong \operatorname{End}_{\bar{R}}(\bar{I})$. Moreover, we can assume that $\operatorname{Ann}_{R}(I)=P_{1} \cap \ldots \cap P_{s}$ for some $s \leqslant t$, from which it follows that the minimal primes of $\bar{R}$ are $\bar{P}_{1}, \ldots, \bar{P}_{s}$, where $\bar{P}_{i}=P_{i} / \operatorname{Ann}_{R}(I)$. Then $\bar{R} / \bar{P}_{i} \cong R / P_{i}=R_{i}$ has local integral closure $\bar{R}_{i}$ in its field of fractions $Q_{i}$ for each index $i, 1 \leqslant i \leqslant s$. Thus, replacing $I$ by $\bar{I}$ and $R$ by $\bar{R}$, we can assume that $I$ is an indecomposable regular ideal of $R$.

Let $R^{\prime}=\operatorname{End}_{R}(I)$, an overring of $R$. For each index $i$, let $\tilde{P}_{i}$ be the kernel of the natural map from $\tilde{R}$ onto $\tilde{R}_{i}$, so that $\tilde{P}_{1}, \ldots, \tilde{P}_{t}$ are the distinct minimal primes of $\tilde{R}$. Moreover, their contractions to $R$ yield the distinct minimal primes of $R$, namely, $P_{i}=R \cap \tilde{P}_{i}$ for each index $i$. Let $P_{i}^{\prime}=R^{\prime} \cap \tilde{P}_{i}$ for each index $i$; then the ideals $P_{1}^{\prime}, \ldots, P_{t}^{\prime}$ must be the distinct minimal primes of $R^{\prime}$. Now each $\tilde{P}_{i}$ is contained in only one maximal ideal, since $\tilde{R} / \tilde{P}_{i}=\tilde{R}_{i}$ is assumed to be local. Since $\tilde{R}$ is integral over $R^{\prime}$, by "going-up" each minimal prime ideal of $R^{\prime}$ is contained in a unique maximal ideal as well. If $M^{\prime}$ is an arbitrary maximal ideal of $R^{\prime}$, we can number the minimal primes of $R^{\prime}$ so that $P_{1}^{\prime}, \ldots, P_{r}^{\prime} \subseteq M^{\prime}$ while $P_{r+1}^{\prime}, \ldots, P_{t}^{\prime} \nsubseteq M^{\prime}$, for some integer $r, 1 \leqslant r \leqslant t$. Then $V\left(P_{1}^{\prime}\right) \cup \ldots \cup V\left(P_{r}^{\prime}\right)$ is both open and closed in $\operatorname{Spec}\left(R^{\prime}\right)$, because each minimal prime of $R^{\prime}$ is contained in a unique maximal 
ideal. Since $R^{\prime}$ must be an indecomposable ring, because $I$ is an indecomposable module, it follows that $r=t$, and every minimal prime of $R^{\prime}$ is contained in $M^{\prime}$. Thus, $M^{\prime}$ is the unique maximal ideal of $R^{\prime}$, so that $R^{\prime}$ is local, as claimed.

Since each indecomposable ideal of $R$ has a local endomorphism ring over $R$, by [1. Proposition V.3.4], $R$ has the UDI property.

\section{EXAmples}

In this section, we construct several examples of reduced commutative Noetherian rings which satisfy the UDI property, illustrating both Theorem 2.5 and Theorem 3.6.

The examples of non-local integral domains with the UDI property in [4] all have Krull dimension one; our first example shows that this need not always hold. (Our thanks to Tim Ford for this example.)

Example 4.1. Let $F$ be an algebraically closed field, and consider the ring $F[X, Y]$ of polynomials over $F$ in two indeterminates $X$ and $Y$. Let $S$ be the multiplicatively closed set in $F[X, Y]$ generated by polynomials of the form $X-\alpha$ and $Y-\beta$ for all non-zero elements $\alpha, \beta \in F$, and set $R=S^{-1} F[X, Y]$, a Noetherian domain of Krull dimension two. The only non-principal maximal ideal of $R$ is $M=S^{-1}(X, Y)$, while $R$ has infinitely many principal (height one) maximal ideals. Since $R_{M}=$ $F[X, Y]_{(X, Y)}$ is integrally closed and local, $R$ has the UDI property by Theorems 2.5 and 3.1 .

Non-local non-domains with the UDI property and Krull dimension greater than one are easy to construct. In fact, as the following example demonstrates, there is no bound to the possible Krull dimension of a non-local ring with the UDI property.

Example 4.2. Consider the conductor square

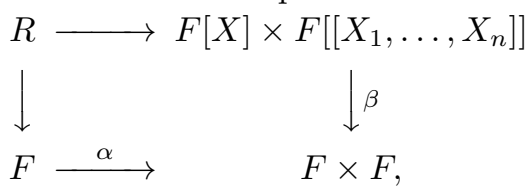

where $F$ is a field, $F[X]$ is a polynomial ring in one indeterminate, and $F\left[\left[X_{1}, \ldots\right.\right.$, $\left.\left.X_{n}\right]\right]$ is a power series ring in $n$ indeterminates for some positive integer $n$. Here $\alpha$ is the diagonal inclusion map $\alpha(r)=(r, r)$ for each $r \in F$, and $\beta$ is the map sending polynomials and power series to their constant terms $\beta(f, g)=\left(f_{0}, g_{0}\right)$. Thus,

$$
R=\left\{(f, g) \in F[X] \times F\left[\left[X_{1}, \ldots, X_{n}\right]\right]: f_{0}=g_{0}\right\}=\beta^{-1}(\operatorname{Im}(\alpha)),
$$

and the conductor $M=(X) \times\left(X_{1}, \ldots, X_{n}\right)$ is the largest common ideal of $R$ and $F[X] \times F\left[\left[X_{1}, \ldots, X_{n}\right]\right]$.

Note that $F[X] \times F\left[\left[X_{1}, \ldots, X_{n}\right]\right]$ is both integrally closed and finitely generated as a module over $R$ (by the idempotents $(1,0)$ and $(0,1)$ ), so that $F[X] \times$ $F\left[\left[X_{1}, \ldots, X_{n}\right]\right]$ is the integral closure of $R$ in their common total ring of quotients $F(X) \times F\left(\left(X_{1}, \ldots, X_{n}\right)\right)$. In the notation of the introduction, $\tilde{R}=F[X] \times$ $F\left[\left[X_{1}, \ldots, X_{n}\right]\right]$ and $Q=F(X) \times F\left(\left(X_{1}, \ldots, X_{n}\right)\right)$. Thus, $R$ is Noetherian [7, Theorem 3.7(i)], because $\tilde{R}$ is Noetherian and finitely generated as a module over $R$. Moreover, $R$ has dimension $n$, the dimension of its integral closure $\tilde{R}$. We also observe that $R$ has no non-trivial idempotents, so that $R$ is indecomposable.

We claim that $M$ is the unique non-principal maximal ideal of $R$. Being the kernel of $\beta$ mapping $R$ onto $F, M$ is a maximal ideal of $R$. If $(X, 0) \in(f, g) R$ 
for some $(f, g) \in M$, then $g=0$ because $(1,0) \notin R$, and hence $(f, g) R \neq M$; that is, $M$ is non-principal. On the other hand, the maximal ideals of $\tilde{R}$ which do not lie over $M$ are of the form $N_{p}=p F[X] \times F\left[\left[X_{1}, \ldots, X_{n}\right]\right]$, where $p \in F[X]$ is a monic irreducible polynomial other than $X$, and one easily checks that $N_{p} \cap R$ is the principal maximal ideal $(p, p(0)) R$. Since every maximal ideal of $R$ is the contraction of a maximal ideal of $\tilde{R}$, the claim follows.

Finally, we claim that $R$ has the UDI property. By Theorem 2.5, it suffices to check that $R_{M}$ has the UDI property. Since $R_{M}$ is a flat $R$-module, by [3, Lemma 1.1.6], localizing the above conductor square at $M$ gives a conductor square

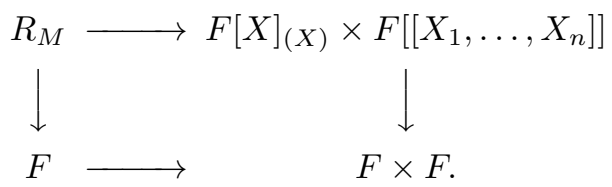

$R_{M}$ remains indecomposable, and $\tilde{R}_{M}=F[X]_{(X)} \times F[X, Y]$ is a product of local rings, so that $R_{M}$ has the UDI property by Theorem $[3.6$ (3).

We conclude this section with examples illustrating cases (2) and (3) of Theorem 3.6.

Example 4.3. Consider the conductor square

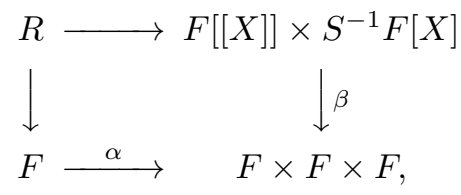

where $F$ is a field, $F[[X]]$ is a power series ring in one indeterminate, $F[X]$ is a polynomial ring in one variable, and $S=F[X]-((X) \cup(X-1))$ is a multiplicatively closed subset of $F[X]$, so that $S^{-1} F[X]$ has exactly two maximal ideals $(X)$ and $(X-1)$. Again $\alpha$ is the diagonal inclusion, while $\beta(f, g)=(f(0), g(0), g(1))$ for $f \in F[[X]]$ and $g \in S^{-1} F[X]$. Thus,

$$
\left.R=\left\{(f, g) \in F[[X]] \times S^{-1} F[X]: f(0)=g(0)=g(1)\right)\right\},
$$

and the conductor is $M=(X) \times((X) \cap(X-1))$.

As in Example 4.2, note that $R$ is an indecomposable, reduced, commutative, Noetherian ring, with integral closure $\tilde{R}=F[[X]] \times S^{-1} F[X]$. The only maximal ideals of $\tilde{R}$ are $F[[X]] \times(X), F[[X]] \times(X-1)$, and $(X) \times S^{-1} F[X]$, and these maximal ideals all contract to $M$ in $R$, so that $R$ is local. Moreover, $\tilde{R}_{1}=F[[X]]$ is local, while $\tilde{R}_{2}=S^{-1} F[X]$ has maximal ideals $(X)$ and $(X-1)$, where $M \nsubseteq F[[X]] \times(X)^{2}$ and $M \nsubseteq F[[X]] \times(X-1)^{2}$, and $R / M=F=S^{-1} F[X] /(X)=S^{-1} F[X] /(X-1)$. Thus, $R$ has the UDI property by Theorem 3.6(2).

Example 4.4. Consider the conductor square

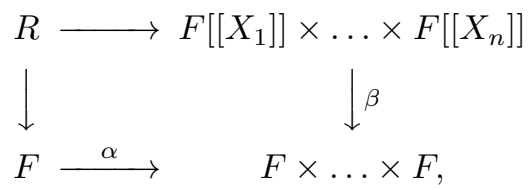

where again $F$ is a field, each $F\left[\left[X_{i}\right]\right]$ is a power series ring in one indeterminate, $\alpha$ is the diagonal inclusion, and $\beta$ maps each $n$-tuple of power series to the $n$-tuple 
of constant terms. In this case,

$$
R=\left\{\left(f_{1}, \ldots, f_{n}\right) \in F\left[\left[X_{1}\right]\right] \times \ldots \times F\left[\left[X_{n}\right]\right]: f_{1}(0)=\cdots=f_{n}(0)\right\},
$$

and the conductor is $M=\left(X_{1}\right) \times \ldots \times\left(X_{n}\right)$.

Also as in Example 4.2, we observe that $R$ is an indecomposable, reduced, commutative, Noetherian ring. Moreover, $M$ is the only maximal ideal of $R$, so that $R$ is local. The integral closure of $R$ is the $\operatorname{ring} \tilde{R}=F\left[\left[X_{1}\right]\right] \times \ldots \times F\left[\left[X_{n}\right]\right]$, a product of local rings, so by Theorem $3.6(3), R$ has the UDI property. Note that $\tilde{R}$ has exactly $n$ distinct maximal ideals, where the choice of $n \geq 1$ is arbitrary in this example, so there is no bound to the number of maximal ideals possible for the integral closure of a local ring with the UDI property in Theorem 3.6, unlike the situation for integral domains in [4, Theorem 3.2].

\section{ACKNowledgement}

The authors would like to thank the anonymous referee, whose numerous suggestions greatly improved the exposition of this paper, especially in the second section.

\section{REFERENCES}

[1] H. Bass, Algebraic K-theory, W. A. Benjamin, New York, 1968. MR0249491 (40:2736)

[2] E. G. Evans, Krull Schimdt and Cancellation over Local Rings, Pac. J. Math. 46 (1973), 115-121. MR0323815 (48:2170)

[3] M. Fontana, J. A. Huckaba, I. J. Papick, Prüfer Domains, Marcel Dekker, New York, 1997. MR 1413297 (98d:13021)

[4] P. Goeters, B. Olberding, Unique Decomposition into Ideals for Noetherian Domains, J. Pure Appl. Algebra 165 (2001), 169-182. MR.1865965 (2002i:13023)

[5] T.W. Hungerford, Algebra, Graduate Texts in Mathematics 73, Springer, Berlin, 1980. MR600654 (82a:00006)

[6] B. R. McDonald, Linear Algebra Over Commutative Rings, Marcel Dekker, Inc., New York, 1984. MR769104 (86d:13008)

[7] H. Matsumura. Commutative Ring Theory, Cambridge University Press, Cambridge, 1986. MR:879273 (88h:13001)

[8] J.-P. Serre, Local Fields, Springer-Verlag, Berlin, 1979. MR554237 (82e:12016)

Department of Mathematics, Computer Science and Statistics, Ohio State UniverSiTy, Lima, OHIO 45804

Department of Mathematical Sciences, Florida Atlantic University, Boca Raton, FLORIDA 33431 